\title{
lieuxdits \#7
}

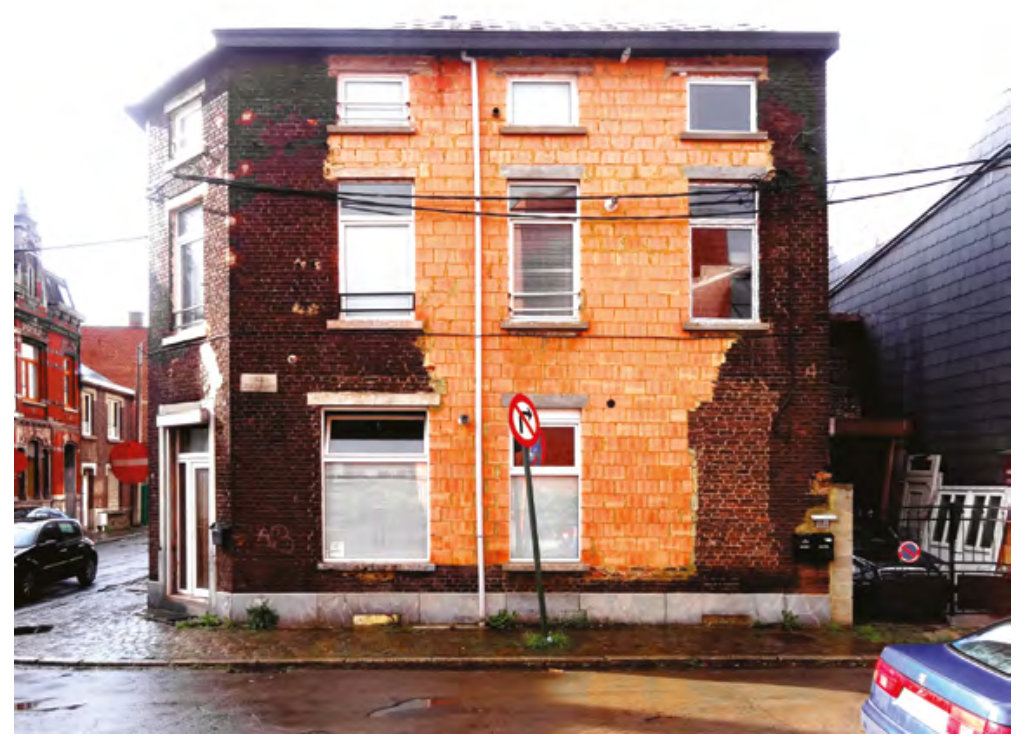

Référence bibliographique :

Corentin Haubruge et Guillaume Vanneste, "École buissonnière", lieuxdits\#7, mars 2014, pp.20-23.

\section{La revue lieuxdits}

Faculté d'architecture, d'ingénierie architecturale, d'urbanisme (LOCI)

Univesrité catholique de Louvain (UCL).

Éditeur responsable : Jean-Paul Verleyen, place des Sciences, 1 - 1348 Louvain-la-Neuve

Comité de rédaction : Martin Buysse, Damien Claeys, Gauthier Coton,

Jean-Philippe De Visscher, Guillaume Vanneste, Jean-Paul Verleyen

Conception graphique : Nicolas Lorent

Impression : école d'imprimerie Saint-Luc Tournai

\section{(C) (i) (8) $\Theta$}




\title{
École buissonnière ${ }^{1}$
}

\author{
Corentin Haubruge et Guillaume Vanneste
}

L'UCL est sur le point d'acquérir un nouveau bâtiment au centre de Tournai afin d'y reloger sa Faculté d'architecture, actuellement sise à Ramegnies-Chin et à l'étroit dans ses locaux.

Dès la rentrée 2015-2016, les étudiants de l'UCL pourront donc suivre leurs cours au sein des $7000 \mathrm{~m}^{2}$ des bâtiments détenus aujourd' hui par le Groupe $3 S I^{2}$

\section{Genèse}

À quelques mois de remplir nos cartons de livres et de vestiges de maquettes, avant de voir débarquer un convoi de camions déménageurs embarquer anonymement des tonnes d'objets de mémoire, avant de s'installer dans de nouveaux murs, revenons sur le contexte du départ et ce qu'il a provoqué au sein de l'école.

Entre l'excitation face au changement et la nostalgie de devoir quitter d'anciens lieux chargés d'une histoire longue, complexe et surtout commune à plusieurs générations d'architectes. Atavismes et opportunités. Ce texte n'ambitionne pas de retracer le parcours exhaustif de l'institution ou de rédiger une exégèse historique, mais simplement de dresser le portrait et de faire l'état des lieux de ce moment zéro qui précède le déménagement.

Des questions reviennent, lancinantes, ou apparaissent, encore inexplorées. Quels devraient être les lieux pour l'enseignement de l'architecture ? Peut-on apprendre aussi bien l'architecture dans la banlieue française, dans un couvent isolé du Tournaisis, au fond de la Suisse italienne ou au cœur de la Cité ? Pourquoi le lieu où l'on enseigne importerait plus en architecture que pour une autre discipline ? Ou comment apprendre la ville si on ne la voit pas, si on n'y est pas ? Un "campus" tel qu'a pu l'être Passy-Froyennes, n'est-il pourtant pas déjà pensé comme une petite cité en soi ?

1 - Références aux écoles clandestines dites "buissonnières" tenues dans les campagnes par les luthériens dans les années 1550 .

2- Extrait du communiqué officiel UCL du 25 juin 2013 (http://www. uclouvain.be/446035.html).

3- D. Maurage,

Passy-Froyennes: 1904-2004. aux projets d'étudiants, aux bruits de couloirs des "pros et contras", à des commissions de recherche de bâtiments, à une exposition en centre-ville, comme autant d'essais de laboratoire, destinés à tester ou à jauger ce que sera la vraie expérience, grandeur nature. A cette occasion, l'école met à l'épreuve sa posture, son enseignement de l'architecture.

\section{État des lieux}

Cet enseignement trouve ses fondements en 1877, année de la construction de l'école Saint-Luc à Tournai, la seconde du genre en Belgique après celle de Gand. Initialement école de dessin située à la rue du Four-chapitre, puis rue du Curé Notre-Dame, elle dispense en 1887 des cours de menuiserie et de sculpture sur bois, avant de déménager rue des Clairisses, et d'intégrer les cours de peinture décorative et de sculpture sur pierre.

Après un passage en 1896 à la rue Tête d'or, l'année 1911 marque un tournant, puisque tout l'enseignement professionnel et artistique de l'école se base désormais sur l'architecture. À cette époque, la "pédagogie artistique originale est développée prônant la maitrise parfaite et le dévouement de l'artisan à son métier et l'expression d'un message socioreligieux émanant de l'architecture contribuant à former la personnalité de l'élève" ${ }^{3}$

Suite aux bombardements de mai 1940 , les différentes sections se retrouvent éclatées dans la ville, l'architecture s'établissant rue du Chambge. II faut attendre l'après-guerre et le rapatriement en France du collège parisien de PassyFroyennes pour que l'école déménage sur le site actuel, en 1960.

Cet édifice néo-gothique monumental créé en 1904 par les frères de Passy- 
Paris, en réaction à la Loi Combes garde la trace de ce passage de frontière, comme en témoignent les ornementations de tous styles reprenant le motif du lys de France.

De cette école et de son esprit, que subsiste-t-il finalement ? La nationalité majoritaire de ses hôtes $(85 \%$ des étudiants sont français) et l'architecture sans doute, le temps ayant eu raison des usages initiaux.

Témoin de cette évolution, un géant d'antan, le château d'eau, est aujourd'hui décapité. N'occultons pas les progrès de l'hydraulique urbaine qui ont eu, entre autres, raison de ce totem faisant écho au clocher de la chapelle, mais retenons-en la part symbolique. Celui de l'autarcie monacale, à l'usage des étudiants de Paris qui débarquaient en train au beau milieu du site, logés en pension la semaine, rapatriés le weekend. Le musée, le gymnase, le terrain de tennis, la piscine, la boulangerie, le lavoir, les potagers, et dans une certaine mesure le pensionnat ont fini par disparaitre.

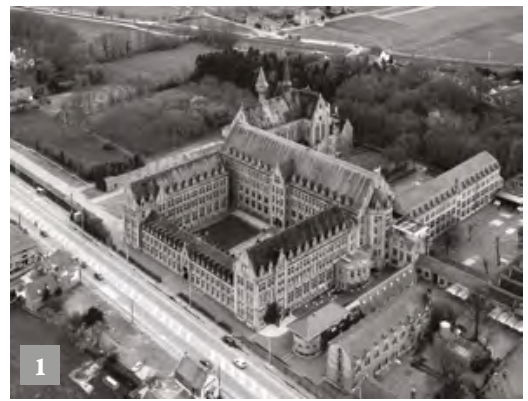

Aujourd'hui, l'école est devenue un lieu de passage, d'apprentissage et d'échange ponctuel, un milieu attractifrépulsif de "learn \& go". Ce retournement des usages, de l'oasis au désert, est en grande partie le résultat de l'individualisation des moyens de déplacements.

L'histoire est bien connue, symbole moderne de progrès et de liberté ${ }^{5}$, l'automobile est devenue une nuisance. La pollution, la sur-mobilité menant à l'immobilité des réseaux saturés, le stationnement des centres urbains entre autres sont autant reproches qui lui sont adressés.

Conte ou cauchemar, la voiture n'en reste pas moins une des conditions actuelles de la survie de l'école de LOCI à Froyennes. Le parking inspire et expire au rythme de l'horaire de l'école, drainant les flux d'étudiants venant pratiquer l'architecture sous une règle de composition - la symétrie - d'un autre temps, voire dans des espaces complètement réappropriés - la ferme de l'école pour les années de Master.

Tout aussi dépassée soit-elle, l'école délivre chaque année son lot de projets, dont la qualité globale peut être jugée inversement proportionnelle à celle de la qualité des lieux d'un point de vue organisationnel et logistique. Fruit d'une cohérence et d'une générosité à mettre au crédit du corps enseignant, ainsi que de la culture débrouillarde des étudiants, ce miracle local est régulièrement souligné, par les professeurs invités notamment. Un constat que nous pouvons encore poser au terme de cette année, qui livre un large éventail de visions et de positions affirmées dans divers ateliers répondant à la question "quelle école d'architecture idéale à Tournai ?"

À notre connaissance cependant, aucun n'étudiant n'avait jeté son dévolu sur le site retenu finalement par les autorités de I'UCL. L'idéal de l'école d'architecture ne semblait donc pas s'imaginer sur ce site, le dénommé "Cofidis", ou "3 Suisses". D'autres lieux ont mieux incarné le rêve d'étudiants pour les générations à venir la plaine des manœuvres, la friche et le rempart de la caserne Saint-Jean, les bords de l'Escaut et ses innombrables dilatations urbaines, les abords de la gare et des friches ferroviaires, les quais jouxtant le Pont des Trous, la maison des Prêtres, le GB (seul édifice à caractère moderne au centre-ville), les carrières, les friches industrielles, les édifices et îlots à rénover...
L'autarcie sous la symétrie de pierre et de hys (photo issue de la galerie de photos du site du pensionnat de Saint-Lu Froyennes:

http://www.pslt.be/galerie-de-photos)

Tournai, ville pétrifiée, réalisation de Guilhem Chuilon, étudiant Master 2. LOCITRN, 2012-2013.

5- "Je crois que l'automobile est aujourd' hui l'équivalent assez exact des grandes cathédrales gothiques: je veux dire une grande création d'époque, conçue passionnément par des artistes inconnus, consommée dans son image, sinon dan son usage, par un peuple entier qui s'approprie en elle un objet parfaitement magique."

In R. BARTHES, La nowvelle Citroën extrait de Mythologies, 1957 
Fort de ce matériel, une exposition de travaux d'étudiants a habillé l'office du tourisme le temps de l'été 2013, diffusant au cœur de ville une série de projets réalisés sur des sites de Tournai. Opportunité culturelle et politique, l'évènement se veut la vitrine d'un savoir-penser et d'un savoir-faire transversaux, du territoire au détail.

Une visibilité et un moyen de communiquer avec l'habitant et le passant, des amateurs aux acteurs. Une mission de transmission et d'éveil, impossible sur le site actuel de Froyennes, qui rend compte du contenu (les travaux réalisés) et du statut universitaire récent de l'école.

Cette exposition peut être perçue comme un premier jalon du retour intramuros. Un orteil en ville au pied de la cathédrale. Une première pierre posée, qui en appelle d'autres, partie visible de la dynamique en cours précédant un tournant historique.

\section{Cas d'écoles}

D'autres écoles d'architecture ont déménagé ailleurs en Europe. Du campus à la ville. Ou l'inverse. L'école de Porto pétrifie un renouveau de l'architecture portugaise. La faculté d'architecture de Lausanne est la dernière à rejoindre le campus de I'EPFL. L'école d'architecture de Nantes ancre une opération de redéveloppement urbain sur une friche industrielle au cœur de l'agglomération. Lyon décentre son école nationale d'architecture dans sa périphérie nord. Et beaucoup d'autres.

Choix libres ou contraintes externes. Sans vouloir tirer de conclusions directes, on peut simplement chercher à comprendre les choix opérés et faire un tour d'horizon des solutions choisies.

À Porto, en 1987 lorsque l'école des Beaux-Arts devient trop petite pour accueillir la masse grandissante des étudiants en architecture, la construction de l'école d'architecture est confiée à I'architecte Alvaro Siza. La nouvelle implantation de l'école, maintenant autonome, correspond aussi à une sorte de reconnaissance d'un modernisme régionaliste dans le nord du Portugal avec pour chefs de file, Alvaro Siza puis Souto de Moura.

L'expérience portugaise : un atelier en plein air au centre de la pédagogie

En plein cœur de l'île de Nantes, l'école d'architecture est construite, au sein du plan guide d'Alexandre Chemetov qui définit la reconversion de cette grande friche industrielle. Sint-Lucas à Gand, grand sœur historique de Tournai, a récemment fait construire des ateliers pour la branche arts visuels par l'architecte Xaveer de Geyter, offrant une certaine visibilité, appréciée ou non, dans le centre-ville.

Le pari est inverse à Lyon en 1982, à Lille en 1977 ou encore à Lausanne qui rejoint en 2000 le reste du campus des années 1980. Ces écoles nationales ou fédérales d'architecture déménagent sur de grands campus dans des territoires nouveaux et souvent périphériques. À ce moment-là, Vaux-en-Velin, Villeneuve-d'Ascq et Ecublens étaient ces nouveaux territoires où les questions de la ville contemporaine se posaient et auxquelles l'université a cherché à montrer par sa présence son implication dans ces questions.

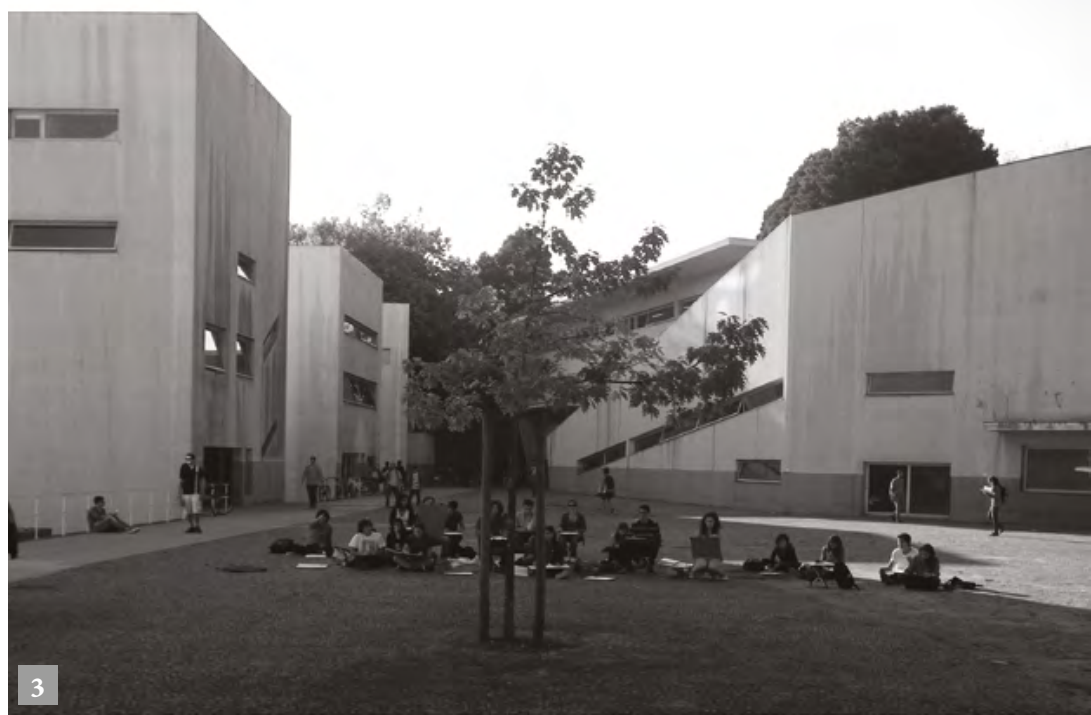


Ces cas d'école, outsiders, ont en commun une situation singulière. En marge de la ville, du reste de l'université, trainant le pied sur les trottoirs quand celle-ci construit en plein champ, s'éloignant quand les autres institutions se concentrent. Comme si le champ disciplinaire dispensé dans ces facultés les amenaient toutes à se positionner, à chercher une manière d'apporter une contribution à la ville, de dire quelque chose de la cité à la cité.

\section{Essuyer les plâtres}

Comment transformer un espace déjà fonctionnel (ce qui semble être démontré) mais finalement peu visible bien que situé en ville, et qui semble être le résultat de compromis en une réelle assise pour un nouveau départ de l'école?

Aujourd'hui, le choix de la faculté en ville est une certitude. Le lieu et la date sont fixés, la crémaillère attend d'être pendue. Mais tout reste à faire.

Qu'attendre d'une institution comme une école d'architecture dans la ville ? Six cents élèves en plein cœur de la cité aux cinq clochers seront plus qu'à même de susciter à eux seuls des réactions, économiques, sociales, artistiques ou culturelles. A contrario, la ville autour de l'école est un agent, un stimulus du développement d'une pensée de l'architecture. Vivre au cœur de faits urbains c'est voir et donc penser la ville chaque jour. C'est bien là le rôle des architectes.
Mais suffira-t-il de poser nos livres et crayons, de brancher quelques prises, de plaquer un peu de plâtre pour faire naître une école d'architecture, par notre seule présence?

Comme les exemples de nos voisins européens, l'occasion de concevoir une nouvelle faculté d'architecture, au-delà de la portée politique de l'évènement, ne doit-elle pas faire montre d'un caractère exemplaire dans ce champ ? Pourrait-on imaginer qu'un architecte propose un projet démontrant ce que pourrait être une école d'architecture dans cette ville réticente au renouvellement contemporain ? Ou entamer une expérimentation maitrisée en offrant la possibilité à de jeunes architectes de réaliser le projet de rénovation du nouveau bâtiment?

Les modes opératoires sont multiples, l'essentiel étant finalement que l'hypothèse formulée repose sur des valeurs, et pas seulement sur un programme, un métré ou un cahier des charges. Que le projet présente une cohérence entre la pédagogie et l'espace de l'école où le savoir serait au service du faire, avec comme pièce centrale l'atelier de projet. Que le projet porte une vision d'école comme vision du monde. C'est la prise de position que doit assumer l'université, une prise de position à la fois formelle, projectuelle et politique. Espérons que Tournai également attende de sa nouvelle école d'architecture qu'elle soit aussi de l'architecture.

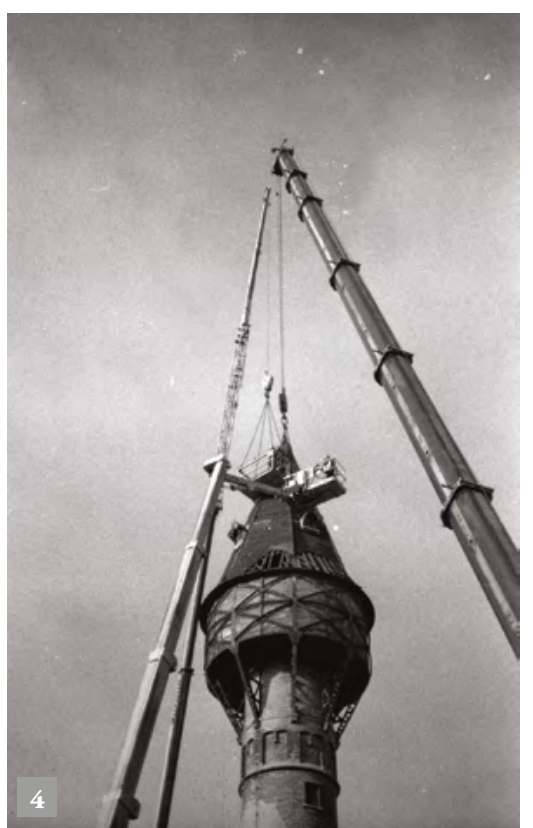

1- Démontage du chateau d'eau à Sain Luc Froyennes. (photo: Timothée Lune photoclub LOCITRN) 